Z. klin. Chem. u. klin. Biochem.

8. Jg., S. $465-468$, September 1970

\title{
Zum Einfluß von Barbital-Natrium auf die Proteinsynthese der Rattenleber
}

\author{
Von H. KRÖNER, K.-H. RudORFF und W. StAIB
}

Institut für Plyysiologische Chemie der Universität Düsseldorf (Direktor: Prof. Dr. S. Hollmann)

(Eingegangen am 13. April 1970)

Frühete Untersuchungen ergaben eine akute Hemmung der Protein- und RNA-Synthese in der Rattenleber nach Gabe von BarbitalNatrium in vivo. Auf Grund dieser Hemmung wurden Enzyminduktionen und die Cortisolgluconcogenese gestört. Auf die Proteinsynthese im zellfreien System in vitro hat die vorherige Gabe von Barbital-Natrium in vivo kcinen sicher nachweisbaren Einfluß. Die Hemmwirkung auf die Proteinsynthese in vivo wird teilweise aufgehoben, wenn Barbital-Natrium in neutralisierter Lösung injiziert wird. Eine wäßrige Lösung von Natriumcarbonat und -bicarbonat von gleichem pH-Wert und gleichem Basenüberschuß wie die Barbital-Natrium-Lösung bewirkt eine Hemmung der Proteinsynthese in vivo. Der Einfluß der relativ geringen pH-Verschiebung auf die Proteinsynthese wird an der isoliert perfundierten Rattenleber bestätigt.

\section{The influence of sodium barbital on protein synthesis in rat liver}

Earlier studies showed that protein and RNA synthesis in rat liver are acutely inhibited following the in vivo administration of sodium barbital. As a result of this inhibition, enzyme induction and cortisol-gluconeogenesis are also disturbed. The prior in vivo administration of sodium barbital has no definite effect on protein synthesis in the cell-free system. The inhibitory action on protein synthesis in vivo is partially abolished if the sodium barbital is injected in neutral solution. An aqueous solution of sodium carbonate and sodium bicarbonate with the same $\mathrm{pH}$ and base excess as the sodium barbital solution causes an inhibition of protein synthesis in vivo. The influence of relatively small $\mathrm{pH}$ changes on protein synthesis was confirmed in isolated perfused rat liver.

Viele Untersuchungen über die Regulation des Stoffwechsels am Ganztier müssen in Narkose durchgeführt werden, und sei es nur, um Stressreaktionen zu vermeiden. Für die häufig länger dauernden Narkosen bieten sich die verschiedenen Derivate der Barbitursäure an. Diese Substanzen, meistens injiziert in Form ihrer Natriumsalze, haben aber selbst eine Wirkung auf den Stoffwechsel. Hofert und Boutwell (1) registrieren eine Hemmung des Aminsosäureeinbaues nach Gabe von Nembutal. Peratno und Mitarbeiter (2) vermuten ebenfalls Stoffwechseleffekte der akuten Phenobarbitalgabe. Wir selbst haben eine Hemmung der Protein- und RNA-Synthese durch Barbital-Natrium in der Rattenleber in vivo beschrieben (3). Weitere Untersuchungen haben gezeigt, daß diese Synthese-Hemmung durch Barbital z. B. wirksam wird bei Enzyminduktionen durch Cortisol oder durch Aminosäuregemisch (4). Die Enzyminduktion wird durch zusätzlich gegebenes Barbital-Natrium zu 50\% und mehr gehemmt, aber auch die Cortisolgluconeogenese wird durch die Narkose um die Hälfte vermindert (4).

Zur Erklärung der Synthesehemmung reicht der durch Barbitalnarkose bedingte Abfall der Körpertemperatur nicht aus (5). In der vorliegenden Arbeit wird daher über weitere Untersuchungen zur Ursache des akuten Barbitaleffektes berichtet. Zunächst wird der Einfluß von Barbital auf die Proteinsynthese in einem zellfreien System in vitro untersucht. Ferner wird der Einfluß des alkalischen $\mathrm{pH}$-Wertes sowie des Alkaliüberschusses der Natriumsalzlösung von Barbital auf die Proteinsynthese in vivo und an der isoliert perfundierten Leber beschrieben.

\section{Methodik}

Adrenalektomierte Wistar-Ratten (Firma Brünger, Bokel), 200 bis $300 \mathrm{~g}$ schwer, blieben $18 \mathrm{Stdn}$. vor der Tötung nüchtern. Zu verschiedenen Zeiten vor der Tötung erhielt ein Teil der Tiere eine einmalige Injektion von Barbital-Natrium $150 \mathrm{mg} / \mathrm{kg}$ i. p. In Äthernarkose wurde die Leber von der Vena portae mit kalter Saccharose-Puffer-Lösung durchströmt und anschließend unter Eiskühlung im Homogenisator von PotTER-ElveHJEM ein Homogenat, ein Teil Leber und vier Teile Saccharose-Pufferlösung, hergestellt. Die Saccharose-Pufferlösung (6) enthielt $0,25 \mathrm{Mol}$ Saccharose, 0,05 Mol Tris/ $\mathrm{HCl}$ Puffer $\mathrm{pH} 7,6 ; 0,025 \mathrm{Mol} \mathrm{KCl}$ und $0,01 \mathrm{Mol} \mathrm{MgCl}_{2}$ im Liter. Das Homogenat wurde zur Entfernung von Kernen und Mitochondrien in der Sorvall Kühlzentrifuge $10 \mathrm{Min}$. bei $800 \mathrm{~g}$ und $20 \mathrm{Min}$. bei $15000 \mathrm{~g}$ zentrifugiert. Vom Uberstand wurde in der Ultrazentrifuge Spinco L2, Fa. Beckman, in einer Std. bei $105000 \mathrm{~g}$ die Mikrosomenfraktion abzentrifugiert. Diese Mikrosomenfraktion wurde in Saccharose-Pufferlösung resuspendiert, und zwar die Mikrosomen von $1 \mathrm{~g}$ Leber in $2,5 \mathrm{ml}$ Pufferlösung.

Das System zum Einbau von Leucin- $\left[1-{ }^{14} \mathrm{C}\right]$ in vitro (7) enthielt in $1 \mathrm{~m} l$ Gesamtrolumen $0,1 \mathrm{~m} l 105000 \mathrm{~g}$ Uberstand und $0,2 \mathrm{~m} l$ Mikrosomenpräparation entsprechend jeweils etwa $2 \mathrm{mg}$ Protein; ferner $1 \mathrm{mM}$ ATP, 0,25 mM GTP, $20 \mathrm{~mm}$ Phosphoenolpyruvat, $20-50 \mu \mathrm{g}$ Pyruvatkinase pro $\mathrm{ml}, 25 \mathrm{~mm} \mathrm{KCl}, 50 \mathrm{mM}$ Tris $\mathrm{pH} 7,6$, $10 \mathrm{mM} \mathrm{MgCl}, 10 \mathrm{~mm}$ Mercaptoaethanol und $0,1 \mu \mathrm{C} / \mathrm{m} l$ Leucin[1-14 C] (spezif. Aktivität $7,25 \mathrm{mC} / \mathrm{mMol}$ ).

Die Inkubation erfolgte $15 \mathrm{Min}$. bei $37^{\circ}$. Es wurden alle vier möglichen Kombinationen von Mikrosomen und Uberstand, von Kontrollen und mit Barbital behandelten Tieren untersucht. Jede Kombination wurde als Dreifach-Bestimmung durchgeführt. Die Reaktion wurde beendet mit $2 \mathrm{ml}$ kalter $0,6 \mathrm{~N} \quad \mathrm{HClO}_{4}$, die $0,02 \mathrm{Mol} / l$ Leucin als 'Träger enthielt. Der Niederschlag wurde abzentrifugiert und einmal mit $2 \mathrm{ml}$ kalter $0,6 \mathrm{~N} \mathrm{HClO}_{4}$ mit Leucin-Zusatz gewaschen. Danach $15 \mathrm{Min}$. bei $70^{\circ}$ mit $2 \mathrm{ml}$ $0,6 \times \mathrm{HClO}_{4}$ extrahiert und nochmals mit kalter $0,6 \mathrm{~N} \mathrm{HClO}_{4}$ gewaschen. Anschließend wurde der Niederschlag in Hyamin aufgelöst und nach Zugabe von Diotol (8) im Flüssigkeitsscintillationszähler Tri-Carb, Fa. Packard, gemessen. Eine Quenchkorrek- 
tur ist meist überflüssig, da der Quench konstant ist und Kontrollmessungen parallel durchgeführt wurden. Entsprechende Lecrwerte, Ansätze wie die Hauptwerte, die jedoch nicht inkubiert, sondern sofort mit Perchlorsäure gestoppt wurden, wurden in Abzug gebracht.

Die Proteinbestimmung erfolgte nach Lowry (9), Eichsubstanz war Serumalbumin vom Rind, reinst, trocken, Fa. Behring. Die RNA wurde nach $15 \mathrm{Min}$ Extraktion mit $1 \mathrm{~N} \mathrm{HClO}_{4}$ durch Messung bei $260 \mathrm{~nm}$ bestimmt, Eichsubstanz war hydrolysierte Kalbsthymus-DNA der Fa. Serva. Den ,, in vitro“ Ansätzen wurde zum Teil Barbital-Natrium zugesetzt, in einer auf $\mathrm{pH} 7,5$ gebrachten Lösung, $1,5 \mathrm{mg} / \mathrm{ml}$ entsprechend $7,3 \mathrm{~mm}$.

Als $\mathrm{Maß}$ für die Proteinsynthese in vivo diente der Einbau von Leucin- $\left[1{ }^{14} \mathrm{C}\right]$ in das Leberprotein. Die Messung der Einbaurate erfolgte wie früher beschrieben (5). Ein Teil der Tiere erhielt eine Std. vor der Tötung eine einmalige Gabe von Barbital-Natrium $150 \mathrm{mg} / \mathrm{kg}$ i. p. Ein zweites Kollektiv erhielt ebenfalls $1 \mathrm{Std}$. vor der Tötung die gleiche Menge Barbital-Natrium, jedoch in einer mit Salzsäure auf $\mathrm{pH} \mathrm{7,2}$ eingestellten Lösung. Ein drittes Kollektiv erhielt pro kg Körpergewicht $10 \mathrm{ml}$ einer Mischung aus $0,075 \mathrm{M}$ Natriumbicarbonat- und $0,075 \mathrm{M}$-carbonatlösung im Verhältnis 2:3. Die Kontrollen erhielten physiol. NaCl-Lösung. Für die Untersuchungen an der isoliert perfundierten Rattenleber wurden Lebern normal ernährter, nicht adrenalektomierter Ratten in Äthernarkose präpariert und mit dem halbsynthetischen Medium nach Schimassek (10) in einer von Miller (11) beschriebenen Apparatur perfundiert (12). Das Perfusionsmedium enthielt in einem Gesamtvolumen von $50 \mathrm{ml} 2 \%$ Serumalbumin vom Rind, reinst (Behringwerke) und Rindererythrocyten entsprechend einem Hämoglobingehalt von $10 \%$. Das entspricht etwa einem Hämatokrit von $25 \%$, der Rest bestand aus Krebs-Ringer-BicarbonatLösung.

Die Leber wurde nach der Operation zunächst 30 Min. perfundiert, anschließend wurden $12 \mathrm{mg}$ Barbital-Natrium in das Perfusionsmedium gegeben, zu einer Endkonzentration von $200 \mu \mathrm{g} / \mathrm{g}$. $30 \mathrm{Min}$. später wurden $2 \mu \mathrm{C}$ Leucin- $\left[1-{ }^{14} \mathrm{C}\right]$ zugesetzt und nach weiteren $30 \mathrm{Min} .2$ Leberlappen entnommen und in flüssigem Stickstoff eingefroren. Die Messung der säurelöslichen und der proteing ebundenen ${ }^{14} \mathrm{C}$-Aktivität erfolgte wie früher beschrieben (5).

\section{Ergebnisse}

Das verwendete System aus Mikrosomenfraktion und $105000 \mathrm{~g}$ Überstand zur Messung der Proteinsynthese in vitro über den Einbau von Leucin- $\left[1-{ }^{14} \mathrm{C}\right]$ ist abhängig von der Energie. Ohne ATP, GTP und Phosphoenolpyruvat findet kein Einbau von Leucin statt. Die gemessene Aktivität dieser Ansätze beträgt etwa 5\% der Aktivität vollständiger Ansätze und entspricht damit den Leerwerten. Die eingebaute Aktivität ist bei den einzelnen Präparationen proportional der zugesetzten Mikrosomen-RNA; halbe Menge Mikrosomen bedeutet halbe Aktivität. $\mathrm{Da}$ die Mikrosomenfraktion nicht gewaschen ist, macht sich ein Fortlassen der Uberstandsfraktion nur mit einer $25 \%$ niedrigeren Ausbeute bemerkbar. Der Zusatz einer Aminosäuremischung ist überflüssig, er ergibt keine höhere Ausbeute.
Ein hemmender Einfluß der Barbitalvorbehandlung in vivo auf die Proteinsynthese in vitro ist nicht festzustellen (Tab. 1). Die Mikrosomenfraktion von Tieren, die 3 Stdn. vor der Tötung Barbital erhielten, scheint etwas aktiver zu sein als die Kontrollen, jedoch ist der Unterschied statistisch nicht gesichert. Der Zusatz von Barbital in vitro in einer Konzentration von $1,5 \mathrm{mg} / \mathrm{ml}$, entsprechend 7,5 mM, bewirkt im Mittel eine Hemmung der Einbaurate um 16,6 $\pm 9,7 \%$. Dabei bleibt zu berücksichtigen, $\mathrm{da} \beta$ die Barbitalkonzentration in vitro die 10-fache derjenigen in vivo ist.

Die Veränderungen der Proteinsynthese in vitro durch geringfügige $\mathrm{pH}-$ Unterschiede bot den äußeren Anlaß, den Einfluß der Alkalinität von Barbital-Natrium-Lösung auf die Proteinsynthese auch in vivo zu untersuchen.

Die Derivate der Barbitursäure sind in Wasser nur relativ wenig löslich und werden daher in Form ihrer Natriumsalze gelöst und injiziert. Diese Natriumsalze reagieren deutlich alkalisch, die verwendete Lösung von Barbital-Natrium 1,5proz., entsprechend $0,073 \mathrm{M}$ hat einen pH-Wert von 10,5.10 ml dieser Lösung werden je $\mathrm{kg}$ Ratte injiziert. Zur Neutralisation der in $10 \mathrm{ml}$ Lösung gelösten $0,73 \mathrm{mVal}$ Barbital-Nátrium auf $\mathrm{pH} 7$ werden $0,45 \mathrm{mVal} \mathrm{HCl}$ benötigt. Eine Lösung, die in $10 \mathrm{ml} \quad 0,45 \mathrm{mMol}$ Natriumcarbonat und $0,3 \mathrm{mMol}$ Natriumcarbonat-Bicarbonat enthält, d.h. eine Mischung aus $0,075 \mathrm{M}$ Natriumcarbonat- und $0,075 \mathrm{M}$ NatriumBicarbonat-Lösung im Verhältnis $3: 2$, hat etwa den gleichen Basenüberschu $\beta$ wie die verwendete Barbital Natriumlösung, ihr pH-Wert beträgt ebenfalls 10,5.

Während eine Std. nach der Injektion von BarbitalNatrium $40 \%$ weniger Leucin- $\left[1-{ }^{14} \mathrm{C}\right]$ in das Leberprotein eingebaut wird als bei den Kontrollen (Tab. 2), beträgt die entsprechende Hemmung durch eine neutralisierte Lösung von Barbital-Natrium nur noch $20 \%$. Diese Hemmung ist jedoch gegenüber den Kontrollen noch statistisch signifikant. Die Injektion der oben beschriebenen $0,075 \mathrm{M}$ Carbonat-Bicarbonatlösung $3: 2$, in einer Dosis von $10 \mathrm{~m} / \mathrm{kg}$ Ratte, bewirkt ebenfalls 30-60 Min. nach der Injektion eirien um 15\% signifikant verminderten Leucineinbau.

In das Protein der isoliert perfundierten Rattenleber wird Leucin- $\left[1{ }^{14} \mathrm{C}\right]$ in etwa gleicher Größenordnung eingebaut wie in das Leberprotein in vivo. Bei einer etwas höheren Ausgangsaktivität von $2 \mu \mathrm{C}$ Leucin-[1-14 C] für $10 \mathrm{~g}$ Leber und $50 \mathrm{ml}$ Perfusionsmedium im Vergleich zu $20 \mu \mathrm{C} / \mathrm{kg}$ Körpergewicht in vivo, ist die Aktivität pro g Leber in vitro etwas höher (Tab. 3). Zu den Unterschieden $z$ wischen den Kontrollen der beiden

Tab. 1

Einbau von Leucin-[1-14 $\mathrm{C}]$ in vitro nach Gabe von Barbital $150 \mathrm{mg} / \mathrm{kg}$ adrenalehtomierte Ratte in vivo

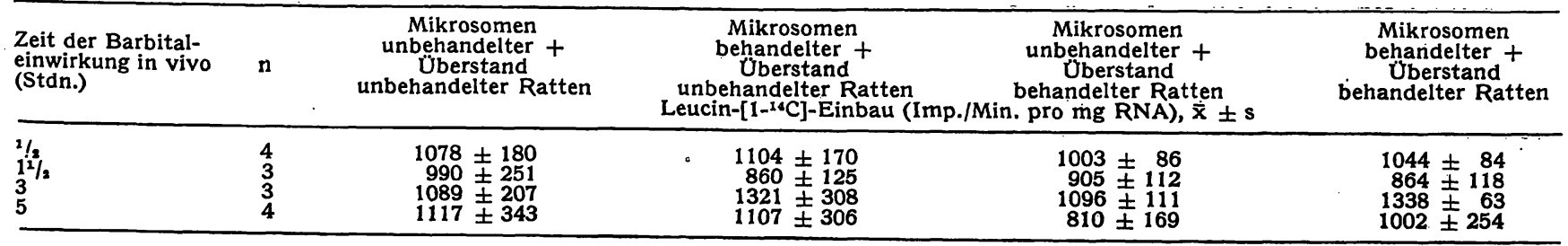


Tab. 2

Einbau von Leucin-[1-14C] in das Leberprotein in vivo $1 \mathrm{Std}$. nach intraperitonealer Injektion von: Barbital-Natrium $150 \mathrm{mg} / \mathrm{kg}$ in physiol. NaCl-Lösung; Barbital-Natrium $150 \mathrm{mg} / \mathrm{kg}$ in neutralisierter (pH 7,2) Lösung; Natriumcarbonat/Bicarbonat $0,075 \mathrm{M}$ im Verhältnis $3: 2,10 \mathrm{ml} / \mathrm{kg}$

\begin{tabular}{|c|c|c|c|c|}
\hline & $\mathbf{n}$ & $\begin{array}{c}\text { Proteing } \\
\text { Imp./Min. pro } \mathrm{g} \text { Leber } \\
\overrightarrow{\overline{\mathrm{x}}} \pm \mathrm{s}\end{array}$ & $\begin{array}{l}\text { CC-Aktivität } \\
\text { Imp./Min. pro mg Protein } \\
\qquad \mathbf{x} \pm \mathbf{s}\end{array}$ & $\begin{array}{c}\text { Säurelösliche }{ }^{\text {"IC }} \text {-Aktivität } \\
\text { Imp./Min. pro g Leber } \\
\overrightarrow{\bar{x}} \pm \mathrm{s}\end{array}$ \\
\hline \multirow{3}{*}{$\begin{array}{l}\text { Kontrollen } \\
\text { Barbital-Natrium } \\
\text { pH unkorrigiert: } 10,5 \\
\text { Kontrollen } \\
\text { Barbital-Natrium } \\
\text { pH korrigiert auf } 7,2 \\
\mathrm{Na}_{2} \mathrm{CO}_{3} / \mathrm{NaHCO}_{3} \mathrm{CO}_{3} \\
\text { pH unkorrigiert: } 10,5\end{array}$} & $\begin{array}{l}4 \\
4\end{array}$ & $\begin{array}{l}58900 \pm 8600 \\
\left.32300 \pm 13600^{2}\right)\end{array}$ & $\begin{array}{l}273 \pm 28 \\
\left.158 \pm 65^{2}\right)\end{array}$ & $\begin{array}{l}8650 \pm 2480 \\
9950 \pm 1480\end{array}$ \\
\hline & $\begin{array}{l}6 \\
5\end{array}$ & $\begin{array}{l}56400 \pm \quad 4300 \\
\left.45600 \pm 7700^{2}\right)\end{array}$ & $\begin{array}{l}270 \pm 22 \\
\left.222 \pm 41^{2}\right)\end{array}$ & $\begin{array}{l}9060 \pm 1310 \\
9910 \pm 2660\end{array}$ \\
\hline & 3 & $\left.48600 \pm 2800^{2}\right)$ & $\left.230 \pm 5^{2}\right)$ & $9620 \pm 1190$ \\
\hline
\end{tabular}

2) $\mathrm{p}<0,05 \quad 2) \mathrm{p}<0,02$

Tab. 3

Einbau von Leucin-[1-14C] in das Protein der isoliert perfundierten Leber. Nach 30 Min. Perfusion Zugabe von Barbital zu einer Endkonzentration von $20 \mathrm{mg} / 100 \mathrm{ml}$ als Natriumsalz bzw. in neutralisierter Lösung. Nach 60 Min. Perfusion Zugabe von Leucin-[1-14C], $2 \mu \mathrm{C}$. Nach weiteren $30 \mathrm{Min}$.

\begin{tabular}{|c|c|c|c|c|}
\hline & $\mathbf{n}$ & $\begin{array}{c}\text { Proteing } \\
\text { Imp./Min. pro g Leber } \\
\overline{\mathbf{x}} \pm \mathbf{s}\end{array}$ & $\begin{array}{l}\text { "C-Aktivität } \\
\text { Imp./Min. pro mg Protein } \\
\qquad \bar{x} \pm s\end{array}$ & $\begin{array}{c}\text { Säurelösliche }{ }^{14} \text { C-Aktivitä } \\
\text { Imp./Min. pro g Leber } \\
\overline{\mathbf{x}} \pm \mathrm{s}\end{array}$ \\
\hline $\begin{array}{l}\text { Kontrollen } \\
\text { Barbital-Na, pH 10,5 } \\
\text { Kontrollen } \\
\text { Barbital-Na, pH 7,2 }\end{array}$ & $\begin{array}{l}4 \\
4 \\
3 \\
3\end{array}$ & $\begin{array}{c}166600 \pm 12800 \\
\left.130300 \pm 17400^{13}\right) \\
74600 \pm 7970 \\
76300 \pm 19800\end{array}$ & $\begin{array}{l}764 \pm 128 \\
644 \pm 138 \\
297 \pm 32 \\
335 \pm 59\end{array}$ & $\begin{array}{l}25000 \pm 2280 \\
35200 \pm 5050 \\
30200 \pm 5850 \\
29000 \pm 5060\end{array}$ \\
\hline
\end{tabular}

1) $\mathrm{p}<0,02$

Serien ist zu sagen, daß die Tiergewichte der ersten Serie bei $200 \mathrm{~g}$, die der zweiten Serie bei $300 \mathrm{~g}$ lagen. Dies dürfte die unterschiedliche Einbaurate nicht ganz erklären. $\mathrm{Da}$ die beiden Versuchsserien in einigem zeitlichen Abstand voneinander durchgeführt wurden, könnten noch andere Unterschiede im Tiermaterial hinzu kommen. Andererseits bestätigt dieser Versuch die Notwendigkeit parallel durchgeführter Kontrollen.

Barbital-Natrium, zu einer Endkonzentration von $20 \mathrm{mg} /$ $100 \mathrm{ml}$ zur Perfusion zugesetzt, vermindert die Einbaurate von Leucin in das Leberprotein um 20\%. Bezogen auf das Lebergewicht ist dieser Wert mit $\mathrm{p}<0,02$ signifikant. Der Anstieg der säurelöslichen ${ }^{14} \mathrm{C}$-Aktivität nach Barbital-Natrium-Zusatz ist gleichermaßen signifikant. Wird das Barbital in einer mit $\mathrm{HCl}$ auf $\mathrm{pH} 7,2$ gebrachten Lösung zugefügt, so läßt sich kein Unterschied in der Leucin-Einbaurate mehr feststellen. Der Zusatz von nicht neutralisierter Barbital-Natrium-Lösung zum Perfusionsmedium in der angegebenen Konzentration macht einen Anstieg des pH-Wertes um 0,1 Einheiten, wie bei mehrfachen Messungen festgestellt wurde.

\section{Diskussion}

Eine Methode, die es gestattet, so unterschiedliche Einflüsse wie z. B. die von Wachstumshormon (13) oder von Tetrachlorkohlenstoff (14) auf die Proteinsynthese der Leberzelle zu analysieren, die Proteinsynthese im zellfreien System in vitro, versagt hier. Während nach Induktion durch mehrmalige Phenobarbitalgabe eine Steigerung des Aminosäureeinbaues in vitro nachzuweisen ist $(15,16)$, ist ein akuter Hemmeffekt an isolierten Mikrosomen nicht festzustellen. Die Lebermikrosomen der mit Barbital $1 / 2$ bis zu 5 Stdn. vorbehandelten Tieren bauen mit gleicher Geschwindigkeit Aminosäuren ein, wie Mikrosomen von Kontrolltieren. Ähnliche Untersuchungen mit Phenobarbital (17) führten zu gleichem Ergebnis.
Die im Mittel geringfügig erniedrigte Einbaurate von Leucin- $\left[1-{ }^{14} \mathrm{C}\right]$ in vitro bei Zusatz von Barbital-Natrium zum zellfreien System in vitro kann mit dem Hemmeffekt in vivo kaum in direkten Zusammenhang gebracht werden. Die Barbitalkonzentration in diesen Versuchen ist zehnmal so hoch wie die im Tierversuch.

Dieses negative Resultat wird verständlicher, berücksichtigt man das Ergebnis unserer weiteren Untersuchungen. Injiziert man eine neutralisierte Lösung von Barbital-Natrium, so wird der Einbau von Leucin$\left[1{ }^{14} \mathrm{C}\right]$ in das Leberprotein in vivo nur etwa halb so stark gehemmt, wie nach Injektion nicht neutralisierter Lösung. Andererseits ist mit einer Lösung von Natriumcarbonat und -bicarbonat von gleichem $\mathrm{pH}$-Wert und gleichem Basenüberschuß wie die Barbital-NatriumLösung eine signifikante Hemmung der Proteinsynthese von $15 \% \mathrm{zu}$ erzielen (Tab. 2). Damit ist die basische Reaktion der Injektionslösung sicher eine wesentliche Ursache für die akute Hemmung der Proteinsynthese. Die Alkalinität der Barbital-Natrium-Lösung als Ursache für die Hemmung der Proteinsynthese geht auch aus den Versuchen an der isoliert perfundierten Leber hervor (Tab. 3). Der bei diesen Versuchen ermittelte Anstieg des $\mathrm{pH}$-Wertes von 0,1 Einheit direkt nach $\mathrm{Zu}$ gabe von Barbital-Natrium dürfte auch für die Versuche in vivo gelten (Tab. 2). Es ist erstaunlich, daß eine so geringe Differenz im $\mathrm{pH}$-Wert, die zudem mit der Zeit abnimmt, übereinstimmend in vitro und in vivo eine Hemmung der Proteinsynthese von 20\% bewirkt. Die $\mathrm{pH}$-Abhängigkeit von Fermentreaktionen ist $z$ war seit langem bekannt (18), jedoch weisen die AktivitätspH-Kurven für isolierte Enzyme meist ein relativ breites Maximum auf (19). Bei der $\mathrm{pH}$-Abhängigkeit eines so komplizierten Systems wie das der Proteinsynthese aus Aminosäuren dürften jedoch verschiedene Faktoren, wie die Veränderung der Michaelis-Konstanten und die Änderung der Dissoziationskonstanten von Substraten und Cofaktoren zusammenwirken. Uber die tatsächliche Bedeutung dieser Phänomene ist noch wenig bekannt(19). 


\section{Literatur}

1. Hofert, J. F. und R. K. Boutwell, Atch. Biochem. Biophysics, 103, 338 (1963). - 2. Peraino, C., C. Lamar JR. und H. C. Pitot, J. biol. Chemistry 244, 2944 (1966). - 3. KRöNER, H., B. GuTENBERGER, S. HollmanN und W. Statb, diese Z. 7, 8 (1969). 4. KröNER, H., H.-E. Bojar, S. HollmanN und W. StaIB, diese Z. 8, 45 (1970). - 5. KRÖNER, H. und W. STAIB, diese Z. 8, 41 (1970). - 6. Lang, N., P. Herritch und C. E. Sekeris, Acta endocr., K'hvn 57, 31 (1968). - 7. SMuckLer, E. A., B. PARThIER und T. Hultin, Biochem. J. 107, 151 (1968). - 8. Herberg, R. J., Analytic Chem. 32, 42 (1960). - 9. Lowry, O. H., N. J. Rosebrough, A. L. Farr und R. J. Randall, J. biol. Chemistty 193, 265 (1951). - 10. Schimassek, H., Biochem. Z. 336, 460
(1963). - 11. MrLLeR, L. L., C. C. Bly, M. L. Watson und W. F. Bale, J. Exper. Med. 94, 431 (1951). - 12. Merers, H. G., J. Flammann, G. Albaum und W. Starb, Biochem. U. 344, 514 (1966): - 13. KoRNER, A., Biochem. J. 92, 449 (1964). - 14. SMUCKLer, E. A. und E. P. BeNDIrT, Biochemistry USA 4, 671 (1965). - 15. KATO, R., W. R. JoNDORF, L. A. LOEB, T. BEN und H. V. GeLborn, Mol. Pharmacol. 2, 171 (1966). - 16. GreIM, H., Hoppe Seyler's Z. physiol, Chem. 349, 1774 (1968). 17. Serfert, G., H. Greim, P. Chïndina, Hoppe Seyler's Z. physiol. Chem. 349, 1179 (1968). - 18. SörENSEN, S. P. L., Biochem. Z. 21, 131 (1909). - 19. NËTrER, H., Theoretische Biochemie, Springer-Verlag, Berlin, Göttingen Heidelberg (1959).

Prof. Dr. W. Staib 4000 Düsseldorf 1 Witzelstr. 111 


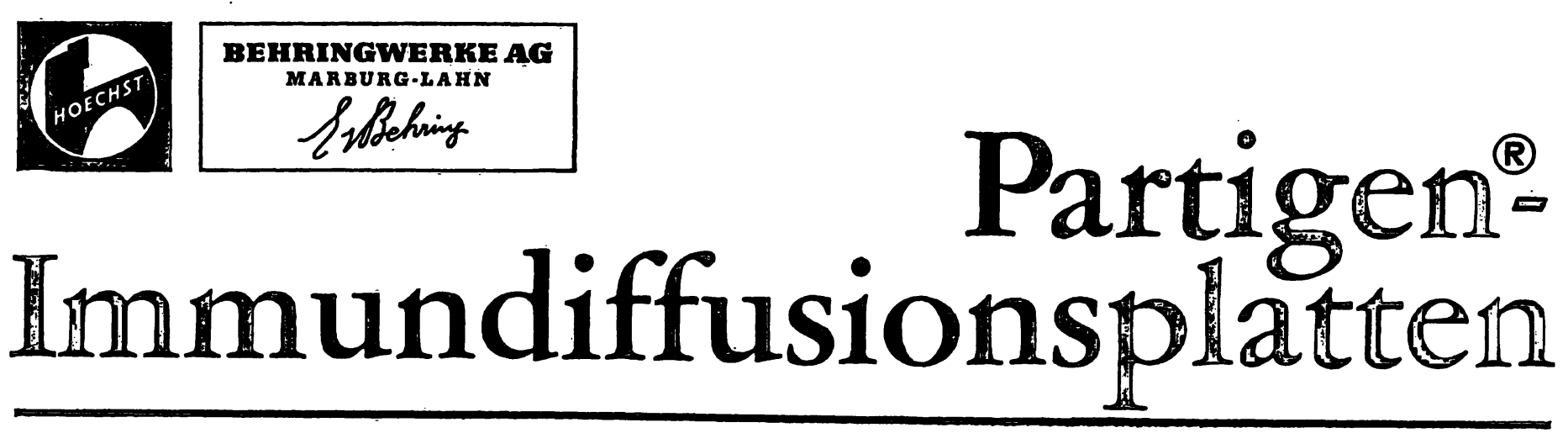

Antiserum-haltige

Agargelplatten Behringwerke für quantitative

Plasmaprotein-Bestimmungen

\section{B63005}
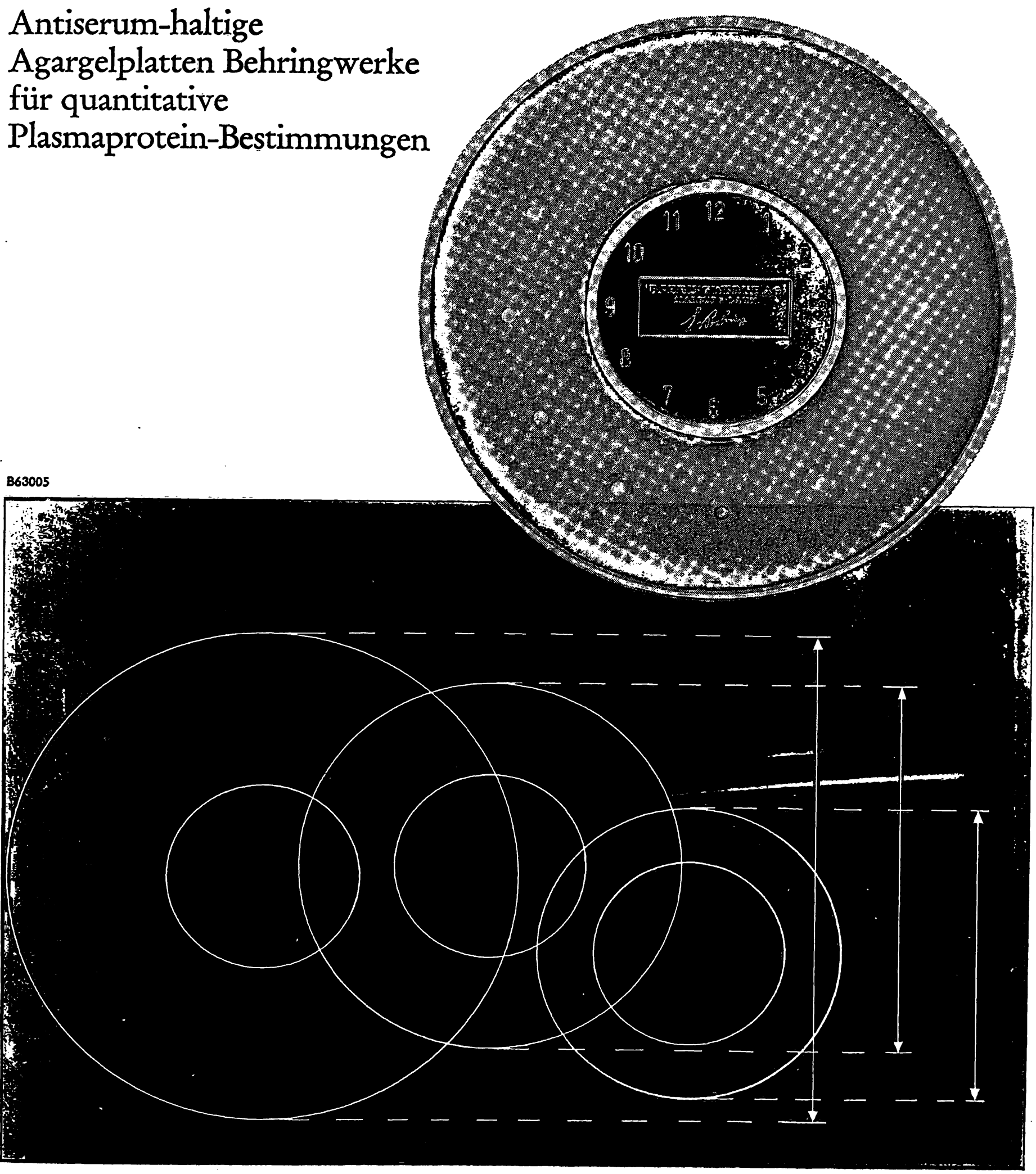


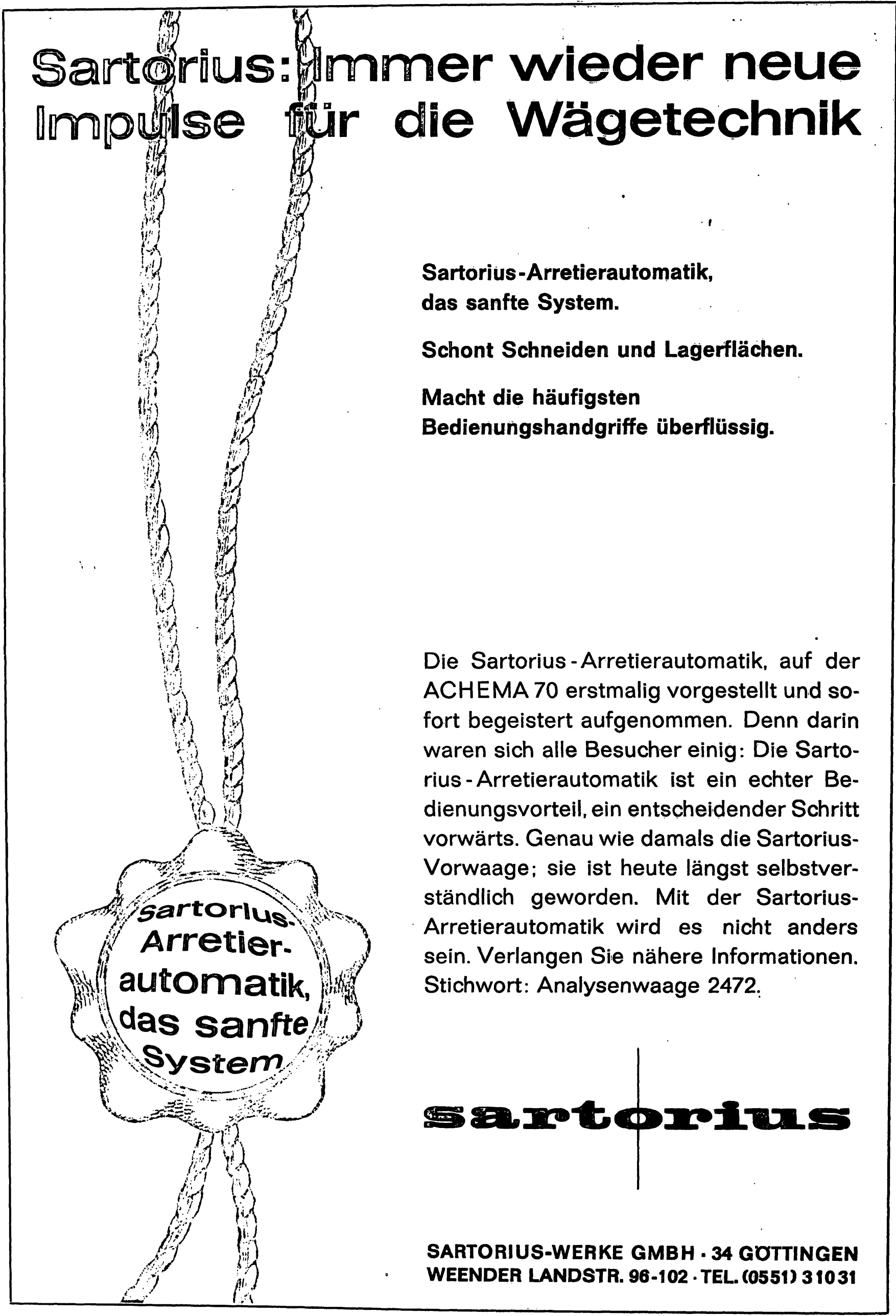

